Diánoia, vol. 15, no. 15, 1969

\title{
LO NORMAL Y LO NORMATIVO
}

Desearía sugerir que la bondad de una cosa es su aptitud para ser gustada y que a su vez esto consiste en que sea de tal tipo que gente normal en circunstancias apropiadas la querría si tuviese una información completa acerca de ella. A primera vista esto puede parecer muy obviamente expuesto al tipo de críticas que los filósofos de la escuela prescriptivista hacen en contra de todas las teorías éticas distintas a la suya propia. "La palabra 'normal' - pueden decir- es ambigua. Puede significar ya sea 'promedio' o 'estándar'. Si usted usa la palabra en el primer sentido, su teoría es plenamente insatisfactoria porque en suma diría que se puede probar la bondad de una cosa simplemente obteniendo el mayor voto en su favor. Y si se usa en el segundo sentido, su teoría es sólo una variante del prescriptivismo, porque el llamar a la gente normal en este sentido es tanto como tomarla como modelo para los hombres en general." No estoy de acuerdo con la versión de normalidad implícita en esta crítica, y creo que la mejor manera de exponer mi punto de vista y al mismo tiempo de aclarar nuestras creencias ordinarias acerca de la bondad es empezar con una comparación entre la bondad y las cualidades secundarias tales como la amarillez.

\section{$\S 1$}

Al criticar un error frecuente entre los filósofos de la moral, al que llamó la falacia naturalista, G. E. Moore declaró en sus Principia Ethica que la bondad era semejante a la amarillez en su indefinibilidad. Su propósito principal al decir esto era el de poner en claro que así como la amarillez, la cualidad perceptible de ciertas cosas materiales, no debe identificarse con la emisión de ciertas vibraciones electromagnéticas, así la bondad no debe identificarse con el placer o con cualquier otra cosa que pudiera tal vez ser un acompañamiento constante o aun una condición necesaria de ella. La observación que yo quiero hacer es diferente. En toda la física moderna subyace una distinción entre cualidades primarias y secundarias. Desafortunadamente a veces se ha dicho de cualidades secundarias tales como color, olor y sabor que no son de ninguna manera cualidades sino sólo sensaciones en nosotros. Afirmaciones a este efecto hechas por Demócrito, Galileo y otras distinguidas personas han conducido a prolongadas y amargas disputas, pero son muy engañosas - de hecho, tan tontas como el error de identificar colores con emisiones de vibraciones. Lo que deberíamos decir es más bien que el ser amarilla una cosa material consiste en aparecer como amarilla a observadores normales en condiciones normales. La cuadratura es una cualidad de 
una cosa material considerada en sí misma, esto es, sin relación a observadores reales o posibles, pero la amarillez es una propiedad relacional. Como dijo Locke en su mejor momento (aunque a veces incurría en la forma errónea de hablar), la amarillez de una cosa material es su poder para producir ciertas experiencias sensoriales en observadores normales. A fin de explicar ese poder dentro de nuestro sistema científico debemos examinar las cualidades primarias de las partes mínimas de la casa material y las características de la radiación que ellas reflejan, pero no podemos identificar propiamente la amarillez con cualquier distribución de las cualidades primarias entre las partes mínimas, porque el poder con el cual se equipara es el poder para producir experiencias y no puede mencionarse sin referencia implícita a los posibles observadores. $Y$ el hecho de que seamos capaces de comunicarnos mutuamente como lo hacemos mediante el uso de la palabra "amarillo" depende de que tengamos constituciones similares y de que seamos afectados de manera similar. Así, si la ceguera total de color fuera tan común entre nosotros como lo es ahora la visión de color, no habría palabras en el uso general en el sentido que ahora le damos a "rojo", "amarillo", "verde" y "azul". Si la pequeña minoría que tuviese lo que nosotros llamamos ahora una visión completa de colores se reuniese, podría tal vez crear un nuevo lenguaje como el nuestro para su propio uso, y aun podría convencer a los otros de que ellos son superiores en cuanto al poder de discernimiento visual. Pero es evidente que la ocurrencia de los adjetivos de colores de cualquier tipo depende en primer lugar de la comunicación social de personas con capacidades similares para la experiencia visual. No quiero decir que la ocurrencia de las palabras valorativas "bueno" y "malo" dependa de la comunicación social de las personas que tienen capacidades similares para la experiencia sensorial, pero sí quiero decir que depende en primer lugar de la comunicación social de las personas que tienen capacidades similares para las actitudes emocionales y prácticas, en particular, tendencias similares para el gusto y el desagrado; y por esta razón sugiero que la bondad podría ser llamada tal vez una cualidad terciaria.

En este respecto no es la única. Considérese, por ejemplo, lo que queremos decir con tales adjetivos como los siguientes cuando se aplican a obras de arte y cosas similares: "agradable", "aborrecible", "gracioso", "tosco", "alegre", "triste", "placentero", "aterrador", "brillante", "aburrido". Claro está que un hombre que dice que cierta película es aterradora no sólo quiere decir que estaba aterrado cuando la vio. De hecho si la viese diez veces, podría estar muy tranquilo en la última ocasión, y sin embargo, ser capaz de reconocer que era aterradora, esto es, que es plausible que aterre a personas normales en circunstancias apropiadas. Digo "personas normales" porque no debemos dejar de llamar aterradora a la película si se encontrara a unas cuantas personas que declararan con evidente sinceridad que no sin- 
tieron ninguna clase de temor al verla, pero que se demostrara en averiguaciones ulteriores que eran incapaces de sentir temor en cualquier situación ordinariamente llamada aterradora. Y digo "en circunstancias apropiadas" para excluir el caso del hombre que la ha visto diez veces y la considera únicamente como una cuestión de técnica, como también el caso de un hombre que la ve por primera vez pero tiene la mente ocupada en algo distinto y no sigue la trama. Sin duda hay con frecuencia lugar para el argumento contra la aplicación del adjetivo "aterrador", pero no creo que la ocurrencia de esos debates haga que cualquiera de nosotros se incline a decir que nada puede ser alguna vez realmente aterrador, esto es, que la palabra deba ser abandonada o no tratada en el futuro sino como la expresión de la emoción momentánea del que habla. A lo más sentiremos que es necesario agregar frases restrictivas, por ejemplo "para niños". Tales cualificaciones son a veces algo así como el desarrollo del uso que hacemos cuando hablamos de sonidos que son demasiado agudos para ser oidos por nosotros, pero que sin embargo pueden ser oídos por los perros. Digo "algo asi como" porque hay, desde luego, grandes diferencias entre los casos. Lo que es importante por el momento es sólo el hecho de que la introducción de palabras como "aterrador" depende de la existencia de capacidades similares de respuesta entre las personas que usan las palabras.

He introducido el término técnico "terciaria" únicamente con el propósito de caracterizar ciertas cualidades que, me parece, están relacionadas con las actitudes humanas de una manera más o menos semejante a aquella en la cual están relacionadas las cualidades secundarias con las sensaciones humanas; y tal vez deberia de poner en claro que las cualidades, a las cuales lo aplico, no son aquellas del tercer tipo que menciona Locke en su Ensayo acerca del entendimiento humano, II, viii, 23. En particular, quiero hacer hincapié en el hecho de que el uso de los adjetivos de cualidades terciarias no podria haberse establecido si no hubiese similitudes considerables entre los hombres con respecto a sus dones afectivos. Pero hay indudablemente diferencias considerables en las maneras en que hablamos de las cualidades secundarias y terciarias y éstas pueden delinearse en su mayor parte como diferencias entre la forma en que llegamos a tener sensaciones y la forma en que llegamos a tener actitudes. Cuando un hombre con visión normal ve el césped fresco a la luz del mediodía le aparece verde. Aquí uso "aparece-verde" con guión para expresar el hecho de que el sujeto tiene ciertas experiencias sensoriales en tanto que dirige sus ojos hacia el césped. No quiero decir que se incline a pensar que el césped es verde; porque podría ser el caso de que cuando lo viese por la noche a la luz de una lámpara roja y no pudiera notar ninguna diferencia de color entre él y la tierra, se percatase, sin embargo, de alguna diferencia en su poder reflectivo y arguyese que esto está relacionado 
con el verdor. Las experiencias sensibles que un hombre tiene mientras ve el césped a la luz del día no son el resultado de ningún razonamiento de su parte. Las tiene en el momento en que abre los ojos y sin ningún examen de evidencia. Hay, tal vez, unas cuantas cosas que nos afectan emocionalmente de esta manera directa; los ruidos fuertes, por ejemplo, nos asustan precisamente cuando no nos los esperamos y no tenemos tiempo de pensar acerca de ellos. Pero muchas de nuestras reacciones hacia las cosas que llamamos agradables, aborrecibles, graciosas, etc., no son de este tipo. Dependen de nuestro estar dispuestos a atender a este o a aquel aspecto de las cosás y por esta razón se puede decir que se fundamentan de manera distinta a la de las sensaciones. Cuando vemos el césped y nos parece-verde, las vibraciones-electro-magnéticas de una longitud de onda entre 5 oo y 575 milimicras afectan nuestros ojos; pero el que tengamos la experiencia sensorial que tenemos no depende de nuestro reconocimiento de ese hecho. Por otro lado, cuando oímos una pieza de música de Mozart y encontramos que es graciosa, nuestra reacción no depende de nuestro reconocimiento de la existencia de ciertos modelos en la música. Para descubrirlos debemos tal vez pasar por cierto entrenamiento, y aun después del entrenamiento podríamos no reparar en ellos si nuestra atención está dirigida a algo distinto. Creemos por tanto que dentro de ciertos limites podemos hacer que la gente reconozca las cualidades terciarias por medio de argumentos, o al menos por educación racional, aunque sabemos que podemos hacer muy poco, si es que algo, por esta vía para mejorar su percepción de las cualidades secundarias. Ésta es una diferencia de extrema importancia; sin embargo, lleva consigo otra que es tal vez aún más importante, una diferencia en el modo en que lidiamos con los desacuerdos.

Cuando encontramos que cierta gente no puede distinguir entre cosas rojas y verdes la llamamos daltoniana. Si como resultado de algún accidente, tal como el daño causado a sus retinas por la radioactividad, el $99 \%$ de la población actual del mundo se tornase incapaz de distinguir las cosas rojas de las verdes, esta gente no tendría dificultad alguna para describirse como daltoniana ya que posee un lenguaje que contiene palabras de colores, y sería capaz de pensarse como anormal en comparación con sus ancestros y consigo misma tal como era cuando aprendió ese lenguaje. ¿Qué deberíamos decir si como resultado de alguna mutación de genes naciesen unas cuantas personas, digamos el $1 \%$ de la población, que pudiesen aparentemente discernir por medio de la visión cosas que al resto de nosotros nos parecieran exactamente iguales? Aun sin instrumentos ópticos tales como los espectroscopios, no deberiamos tener dificultad para idear pruebas a fin de establecer si están fingiendo o no. Tomando, por ejemplo, un número de cartas que afirmaran distinguir por el color, pero que nosotros sólo pudiésemos distinguir por la posición, podríamos 
poner marcas secretas al reverso y después de revolverlas completamente, invitarlos a repetir su separación por colores. Si sin haber dado ocasión para descubrir nuestras marcas secretas hacen la misma división uniformemente una y otra vez, debemos conceder su pretensión. El caso sería aún más fuerte si encontrásemos que la distinción que hicieron corresponde a alguna diferencia de cualidades primarias que podemos establecer de modo independiente, por medio de instrumentos apropiados. Después de esto podríamos tal vez usarlos, como frecuentemente usamos instrumentos científicos, con el propósito de distinguir cosas que nosotros mismos no podemos distinguir únicamente con nuestros sentidos, y probablemente podríamos estar de acuerdo en que el cambio genético que los había producido significa una mejora, puesto que la habilidad para discernir es lo que en general esperamos de nuestros órganos sensoriales. Pero no creo que debamos dejar de considerar a esas personas como anormales, ni tampoco creo que debamos modificar nuestra manera de expresarnos acerca de los colores en alguna manera fundamental después de descubrirlos.

Es algo distinto lo que sucede cuando los hombres están en desacuerdo acerca de las cualidades terciarias. En primer lugar, el desacuerdo no siempre consiste en que un hombre tenga más discernimiento que otro. Puede ser simplemente que uno diga que algo es gracioso y otro que es tosco y sin embargo, hasta donde nos es posible decir, estén de acuerdo con el significado de las palabras que usan. En segundo lugar, aun cuando el desacuerdo de hecho incluye que una de las partes reclame un poder de discernimiento mayor (como cuando por ejemplo el primero dice que ciertas pinturas abstractas están más sutilmente balanceadas que ciertas otras y el segundo no encuentra nada qué escoger entre ellas), no suponemos que la persona que hace más distinciones deba tener la ventaja a condición de que sea coherente. Porque mientras en esos casos la coherencia es condición necesaria de los juicios fundados, ciertamente no es suficiente. Pero en cada desacuerdo acerca de las cualidades terciarias, sea del primer o del segundo tipo, creemos que habría lugar para la persuasión, la cual estaría fuera de lugar en la mayoría de los desacuerdos acerca de cualidades secundarias, y por esta razón los significados de los adjetivos de nuestras cualidades terciarias no están conectadas como los significados de los adjetivos de nuestras cualidades secundarias, con una noción de normalidad meramente estadística. Ciertamente aprendemos a usar las palabras cuando se aplican a casos en donde no hay un desacuerdo importante. ¿De qué otra forma podrían establecerse y perdurar en su uso lingüístico? Pero pronto nos acostumbramos a toparnos con casos donde hay desacuerdo, y al oír argumentos de éstos nos damos cuenta de que alguna gente es más capaz que otra para distinguir los caracteres que son pertinentes en las atribuciones de las variadas cualidades terciarias. Aprendemos esto, sin embargo, úni- 
camente al ser convencidos por tales personas que nos llevan a estar de acuerdo con ellas por sus propias razones, y no por cualquier prueba externa como la que tenemos que aplicar al probar las pretensiones de poderes poco usuales de discernimiento de color. Es verdad que a veces decimos de las personas que consideramos como buenos jueces de las cualidades terciarias que son más sensibles y perceptivas que la gente común; y supongo que estas palabras fueron introducidas primero para sugerir una analogía con el reconocimiento de cualidades secundarias, pero lo que queremos decir con nuestra alabanza es que estas personas son más rápidas para apreciar lo que el resto de nosotros podemos llegar a apreciar. Son más bien como personas que pueden descubrir caras en los rompecabezas o resolver crucigramas más rápido que el resto de nosotros. En sentido estadístico son ciertamente anormales pero no en el sentido de tener poderes de cierto tipo distinto del resto de nosotros. Por el contrario, aprendemos a respetarlos precisamente porque nos permiten hacer lo que ellos han hecho primero. Tal vez algunas veces, cuando nuestro respeto se ha establecido, podemos tener confianza en ellos, esto es, sin haberlos seguido a través de los detalles de las consideraciones que los han convencido. Podemos, por ejemplo, aceptar de un buen matemático que cierta prueba es elegante aunque nosotros la encontrásemos difícil de seguir. Pero aun en este caso pensamos que la prueba produciría una agradable impresión de eficiencia en una persona capaz de captarla; y aceptamos el juicio del especialista porque entendemos y estamos de acuerdo con los juicios del mismo tipo que ha hecho en casos más simples.

Se sigue de todo esto que la concepción de normalidad implícita en nuestra definición de cualidades terciarias no es la pura noción estadística, sino más bien la concepción de alcanzar cierto estándar. Dado este sentido de "normalidad" y la definición de bondad que he sugerido, a saber, que la bondad es una cualidad de ser tal que una persona normal la querría en circunstancias apropiadas si es que está completamente informada. Es bastante natural que en circunstancias apropiadas una descripción de algo como bueno tenga la fuerza social de sugerencia adecuada para escoger cosas de este tipo. Pero no hay nada en lo que he dicho hasta ahora que justifique el punto de vista de que la palabra "bueno" o la palabra "normal" adquiere su sentido del uso en una actividad linguiística especial de recomendación o prescripción la cual debe ser contrastada con la descripción. Qué tipo de normalidad requiere nuestra definición de la palabra "bueno" aún tiene que explicarse detalladamente. Lo que espero mostrar es que el estándar mínimo que debe satisfacer la persona normal de la definición es un estándar de razonabilidad que inevitablemente asumimos en las personas con las cuales sostenemos discusiones morales. Espero también contestar al mismo tiempo la 
objeción de que los seres humanos podrian tal vez pertenecer constitucionalmente a varios grupos de los cuales no se puede esperar que se pongan de acuerdo acerca de lo que es bueno. A veces en cuestiones estéticas nos sentimos inclinados a decir que hay diferencias irreconciliables de gusto y que la única vía sensata es la de abandonar la pretensión de validez inter-personal implicada en el uso de los objetivos de cualidades terciarias en los juicios estéticos; hay escritores de filosofía moral que quieren que hagamos lo mismo en todo el campo del valor. Creo que tales conclusiones no son necesarias.

$$
\$ 2
$$

Muy frecuentemente, tal vez la mayoría de las veces, la palabra "bueno" se aplica a las cosas consideradas como ejemplos de tipos. Decimos, por ejemplo, de cierto objeto que es un buen cuchillo y de otro que es una buena manzana. Ahora bien, obviamente, cuando la palabra "bueno" aparece en posición atributiva antes de un nombre común, no funciona de la misma manera que adjetivos como "cuadrado", "francés", "barbado". De la información de que Carlos I era barbado y también rey podemos concluir sin riesgo que era un rey barbado. Pero de la información de que era un buen esposo y también rey no podemos concluir sin riesgo que era un buen rey. En este respecto, sin embargo, las palabras "bueno" y "malo" no son únicas. De la información que Fido es un pequinés grande, tomada junto con la proposición general de que los pequineses son perros, no podemos inferir propiamente que Fido sea un perro grande. La explicación de estos hechos lingǘsticos es simple. Cuando decimos que Fido es un pequinés grande, queremos decir que es grande para ser pequinés, esto es, más grande que la mayoría de los pequineses; y cuando decimos que Carlos I era un buen esposo, queremos decir que era bueno como esposo, esto es, que poseía las cualidades deseables en un esposo. Aún más, cuando la palabra "bueno" está en posición predicativa como opuesta a la atributiva, a veces debe entenderse como si estuviese unida a un nombre común. Si estoy tratando de comprar un cuchillo y el tendero me dice que el que me atrae es muy bueno, entiendo que habla de él como cuchillo. En estas circunstancias sería bastante absurdo que objetase que es muy ligero y por tanto inutilizable como pisapapel. En cualquiera de los casos tenemos lo que podríamos llamar un uso cualificado o restricto de "bueno". Tal vez el equivalente más claro sea la frase de la forma "bueno como un X", pero hay desde luego muchas otras maneras de dar a entender el sentido. Así a veces Homero describe

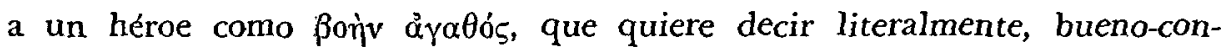
respecto-al-grito-de-guerra; y a veces decimos de un colegial que es bueno 
para la aritmética, aunque no para otras materias. Similarmente a veces decimos que la quinina es buena para curar y prevenir fiebres, cuando, si quisiésemos, podríamos decir que es buena como antipirético. A fin de aclarar lo que está implícito en los usos cualificados de "bueno" consideremos algunos grupos distinguibles de casos; los he escogido para ilustrar algunos puntos que me parecen interesantes aunque no le adjudico importancia alguna al orden en el que aquí los presento.

I) Primero, hay una clase de casos en la cual podemos usar la construcción "bueno como un $\mathrm{X}$ " con un nombre común para artefactos de una cierta clase en la posición final. Quienquiera que entienda el significado de la palabra "cuchillo" sabe que los cuchillos están hechos para cortar y puede, por tanto, estar seguro de que nada es bueno como cuchillo a menos que corte. Esto no quiere decir, sin embargo, que la palabra "bueno" en la combinación "buen cuchillo" signifique lo mismo que "apto para cortar". El profesor Hare sugiere (The Language of Morals, p. 118) que en tales contextos la palabra puede adquirir algo que él llama significado descriptivo como opuesto al valorativo; pero me parece que ésta es una manera engañosa de hablar. Los diccionarios ciertamente enumeran algunos sentidos especiales que "bueno" puede adquirir en contextos especiales tales como "de buenas a primeras" y "bueno por mil libras", pero nunca he oído de un diccionario que tenga una entrada especial para frases como "buen cuchillo", y la razón es bastante clara. Todos esos usos están incluidos de modo adecuado en una entrada general, como la del Oxford English Dictionary, "de cosas que son lo que son llamadas o deberían serlo". Quizá esta forma de palabras no sea tan apropiada en relación a los productos de la naturaleza como lo es en relación a los artefactós, para los cuales ya se presupone un estándar al nombrarlos; pero es al menos un principio prometedor para el trabajo de la definición. Decir que un cuchillo es un buen cuchillo es decir que tiene las cualidades que los hombres normales esperan de un cuchillo, esto es, en este contexto, hombres racionales familiarizados con cuchillos, y una persona que entiende la palabra "cuchillo" puede decir cuáles son esas cualidades sin tener que ir por el mundo haciendo observaciones o persuadiendo a la gente para responder encuestas. A veces damos el mismo sentido de una manera familiar diciendo "ahora si; eso realmente merece llamarse cuchillo" o más simplemente "Eso realmente es un cuchillo". Sin duda, "Este es un buen cuchillo" trae como consecuencia "Esto es lo suficientemente filoso para cortar"; pero ese hecho está explicado lo bastante por las consideraciones separadas de "bueno" y de "cuchillo" dadas en el diccionario. No necesitamos decir que una palabra que es primariamente un término de recomendación ha adquirido, de alguna forma, otro tipo de significado. Por lo contrario, la razón por la cual la observación "Éste es un buen cuchillo" puede ser usa- 
da por un hombre al aconsejar a otro acerca de la elección de un cuchillo es simplemente que "bueno" tiene el sentido arriba mencionado.

2) Un uso muy similar a la construcción "bueno como un $X$ " es aquél en el cual " $\mathrm{X}$ " es remplazado por un nombre común para personas que desempeñan cierto trabajo, por ejemplo, "rey", "plomero", "artista", "matemático", "corredor". Aquí también el uso del nombre común implica ciertas expectativas. De una persona llamada rey se espera que mantenga el orden entre su pueblo, de un plomero se espera que detenga el goteo de las cañerías y así sucesivamente. Hay, sin duda, personas llamadas reyes que causan desorden, asi como hay plomeros que nunca llegan a tiempo para detener el goteo; pero estas personas por lo menos profesan cumplir con las expectativas que acabo de mencionar y tienen su título en virtud de sus profesiones. Decir que cualquiera de ellos es bueno como miembro de su profesión es decir que posee las cualidades que un hombre normal espera encontrar en una persona de esa profesión. Hablo cada vez de lo que un hombre normal espera, porque una persona que usa la palabra "bueno" en tal contexto no está, por un lado, pensando solamente en sus propios deseos particulares ni tampoco, por el otro, tratando de dar cabida a los deseos de los lunáticos, que quizá esperen que los plomeros nunca lleguen a tiempo.

Los dos grupos de casos que hemos considerado hasta ahora son relativamente simples porque el uso de una palabra como "cuchillo" o "plomero" implica expectativas más o menos claras en quien la usa. La mayoría de las veces sabemos lo que queremos de los cuchillos y también lo que queremos de los plomeros. De hecho el significado de los nombres no puede explicarse sin referencia a lo que se supone que hacen los portadores de Ios nombres. Pero aun aquí pueden surgir dificultades de juicio cuando hay varias cualidades distinguibles que queremos. En general queremos que los cuchillos estén afilados para que puedan cortar, pero para algún propósito particular podríamos necesitar un cuchillo que fuese muy fuerte; una hoja de afeitar no tiene utilidad ninguna para cortar piedras. Podria suceder, por tanto, que tuviésemos que balancear una consideración con otra, esto es, de filo de la hoja con la firmeza de la misma, antes de decir cuál de un cierto número de cuchillos es mejor para cierto propósito; para decirlo en otra forma, cuál es mejor como cuchillo de tal y cual tipo especial, esto es, cuchillo para papel, cuchillo para carne, cincel de albañil. De manera similar, cuando los editores de una revista para consumidores deciden juzgar automóviles, deben tener en mente un número de consideraciones diferentes además de la capacidad básica de un automóvil para moverse por la carretera, y es concebible que se encuentren con que no pueden dar ningún juicio claro general de valor, sino que deben conformarse con decir cuáles cualidades deseables se encontrarán en cada marca de coche. No hay nada, sin embargo, en todo esto que nos desanime. ¿Por qué tenemos que asumir que 
siempre será posible llevar a cabo una gradación simple de las cosas que caen bajo un nombre común como "automóvil"? En última instancia, cada hombre que escoge debería decir "Este el mejor coche para mí". Pero aun entonces no debemos suponer que lo que empezó como un intento de hacer un juicio de valor objetivo haya degenerado en la expresión de una mera preferencia subjetiva, porque puede considerarse que "el mejor coche para mi" significa "el coche que satisface mejor mis necesidades especiales"; es importante hacer notar que cuando estamos obligados a hablar de esta forma todavía usamos la palabra "mejor" para significar algo distinto de "lo que de hecho escojo" porque creemos que es posible que un hombre se equivoque en lo que juzga que puede satisfacer sus necesidades. Muy frecuentemente la gente sensata acepta el consejo de otros en esos asuntos y lo sigue aunque no entienda del todo las consideraciones en las que se basa. Por ejemplo, una anciana que no tiene experiencia en asuntos financieros puede muy bien aceptar el consejo del abogado de la familia sobre la mejor manera de invertir su capital para enfrentarse a sus necesidades particulares, porque cree más factible que sea más correcta la opinión del abogado que su propio juicio sin ayuda en ese respecto.

3) Una tercera clase de casos en la cual usamos con frecuencia la construcción "un buen $\mathrm{X}$ " aunque tal vez no tan frecuentemente la construcción "bueno como un $\mathrm{X}$ ", es aquella en la cual ponemos en lugar de " $\mathrm{X}$ " un nombre común de cosas o de personas que pueden ser útiles o de interés para los seres humanos, aunque no creados para ese propósito o nombrados a causa de él. Hablamos, por ejemplo, de una buena manzana, un buen caballo, un buen hermano. Lo interesante de un ejemplo de este tipo es que el entender el significado del nombre común que viene después de "bueno" no es por sí mismo suficiente para poner en claro cualquiera de las cualidades que deberíamos esperar encontrar en las cosas a las cuales se aplica el nombre. Sé, de hecho, que la gente con la que tropiezo en la vida diaria está de acuerdo conmigo en querer manzanas que son comida saludable aunque, aun aquí, hay una posibilidad de escoger entre una variedad de acuerdo con el interés especial del que escoge. También sé que esperan que los caballos sean capaces de llevar jinetes o de jalar cargas, y que quieren que los hermanos sean fieles y generosos de sus hermanos. Puedo, por tanto, decir que una manzana debe de ser por lo menos comible, un buen caballo aquel que es lo suficientemente fuerte para hacer el trabajo y un buen hermano aquel al cual puede acudir un hermano en busca de ayuda y simpatía. Pero no puedo decir propiamente que esas tesis sean verdaderas por definición. Al hacerlas me baso en la información empirica del modelo de vida de mi propia sociedad; porque podría concebirse una sociedad en la cual las manzanas se usaran únicamente para decoración y los caballos sólo para comer y en la cual fuese tabú que los hermanos sostuviesen cualquier conver- 
sación con sus hermanos. Parece, por tanto, que cuando usamos la palabra "bueno" con cualquiera de esos nombres, que sólo estamos tomando como universales, o al menos, como estadísticamente normales, aquellos intereses que encontramos entre la gente que nos rodea. ¿Qué pasaría si nos pusiésemos en contacto con gente de distinta cultura y que tuviésemos que vivir con ella en una sociedad heterogénea? ¿Deberíamos hablar simplemente de un diálogo de sordos? No necesariamente. Nuestra regla general, o más bien costumbre, para el uso de la palabra "bueno" es que su uso no debe sujetarse a los intereses personales o seccionales, sino determinarse por principios aceptables para todos los hombres racionales y, en las circunstancias que he supuesto, podemos preservar esta regla renunciando simplemente a hablar de buenas manzanas, buenos caballos y buenos hermanos, excepto cuando estemos en compañía de personas que son de una misma cultura, y sustituirlas por frases como "buena manzana para comer", "buen caballo para montar" y "buen hermano en el sentido de sociabilidad". Una vez hecho este ajuste, será posible que personas de dos culturas distintas estén de acuerdo, en principio, acerca de cuáles manzanas, caballos y hermanos deban llamarse buenos. La dificultad mayor estaría en el caso último ya que es más fácil permitir diferencias en los hábitos de comer y trabajar que diferencias en la vida familiar. Pero aun aqui la adaptación del lenguaje que sugiero no es imposible mientras las relaciones entre la gente de las dos culturas se limiten a los negocios y a los asuntos públicos. Si las relaciones se estrechasen, tal adaptación ya no sería posible, ya que dos diferentes estándares de comportamiento entre hermanos no pueden sostenerse juntos entre gente unida por matrimonios mixtos.

4) Una cuarta clase de casos en los cuales podríamos encontrar la construcción "Un buen $X$ ", aunque mucho menos frecuentemente "bueno como un X", es aquella en la cual sustituimos " $X$ " por el nombre común de cosas que no tienen un uso obvio para los seres humanos, esto es, "raíz", "guijarro", "cometa", o aun un nombre común de cosas que son generalmente consideradas como dañinas, por ejemplo, "avispa", "huracán". Aquí son posibles varias explicaciones.

a) La cosa llamada buena puede ser concebida tal vez como un órgano o instrumento que sirve a los propóstios de algo con lo que quien habla simpatiza imaginativamente. Podemos decir, por ejemplo, que un árbol tiene una buena raíz, que una araña tiene una buena tela, que un pájaro tiene un buen nido, porque con frecuencia proyectamos nuestras propias nociones de propósito a las descripciones biológicas; por la misma razón, es concebible que digamos que un volcán tiene un buen cráter, pero difícilmente podríamos decir de esta manera que un montón de piedras tiene una buena cima, porque no pensamos en un montón como una cosa con un propósito.

b) Puede ser que quien habla tenga algún interés especial y que asuma 
que sus escuchas comparten o al menos entienden. Puede ser, por ejemplo, que esté jugando a las cabrillas en la playa, y que, cuando dice "ése es un buen guijarro" quiera decir que la cosa indicada es un buen guijarro para usarse en el juego de cabrillas. $O$ nuevamente, puede ser que quien habla esté recolectando veneno de avispa para los fines de algún experimento en bioquímica, y que cuando dice "Ésa es una buena avispa" quiera decir que el insecto es una buena fuente de la sustancia particular que desea. Aquí toda perplejidad desaparece cuando se pone de manifiesto que el nombre común que aparece en la afirmación original debe ser entendido como una abreviación de alguna frase más larga.

c) El que habla puede ser un científico o un maestro de ciencia cuyo sólo interés en la cosa en consideración es que ejemplifica claramente alguna clase natural. Puede, por ejemplo, describir un cometa o un huracán como buenos porque sea especialmente útil para la demostración de los rasgos característicos de los cometas o los huracanes, según sea el caso. Si ésta es la explicación, podemos tomar su afirmación original como la abreviación de un enunciado acerca de que la cosa a la que se refiere es un buen espécimen de su clase. El ejemplo puede entonces ser clasificado como una subvariedad del grupo $b$ ), pero parece requerir especial atención porque lo que decimos de él tiene al menos una similitud superficial con las afirmaciones de Santo Tomás de Aquino acerca de que toda cosa es buena en tanto que realiza su naturaleza perfectamente (Summa Theologiae, I, qu. 5, art. 1). Pero hay en realidad una gran diferencia. El uso de "bueno" al que me he referido conlleva una referencia implícita a un interés humano, no obstante que sea un interés puramente teórico, mientras que Santo Tomás basa su argumento en la tesis metafísica de que cada cosa busca su propia perfección. La conclusión que desearía sacar de los casos que he citado es que de una cosa se puede decir que es un buen $\mathrm{X}$, o bueno como un $\mathrm{X}$, si y sólo si hay alguna cualidad que pueda ser deseada en un $X$ y esta cosa tiene la cualidad. Que tenga la cualidad es un hecho que puede tal vez ser afirmado tanto por una persona que no necesite un $\mathrm{X}$ para ella misma como por quien sí lo necesite. Los catadores de té pueden graduar el té con mayor seguridad que la mayoría de nosotros, pero puede ser gente que nunca beba té en su vida privada. Si estoy en lo cierto, la " $\mathrm{X}$ " en "un buen $\mathrm{X}$ " o "bueno como un X" funciona en forma restrictiva. Tom Smith, diríamos, es un buen plomero pero un mal vecino, y esto implica (en un sentido amplio de "implica"), que, si se desea un plomero por las razones ordinarias por las que la gente desea a los plomeros, será sensato llamar a Tom Smith; mientras que si se está escogiendo dónde vivir y se tienen las necesidades usuales, no será sensato tomar una casa cercana a la de Tom Smith. Supóngase entonces que llegamos al límite y decimos simplemente que algo es una buena cosa. En apariencia hemos levantado todas las restricciones y afirmado incondicionalmente, esto es, sin atender a 
ninguna necesidad o interés en particular, que es una cosa tal que sería del agrado de todas las personas normales. Ahora bien, de hecho nosotros hacemos uso de la frase "una buena cosa" precisamente en este sentido, pero con referencia a estados de cosas más que a sustancias. Diríamos, por ejemplo, que es una buena cosa, o más simplemente, sólo buena, que una gran cantidad de sufrimiento haya sido disminuida por la medicina, o que Stalin fuera remplazado por Krushchev y así sucesivamente. Si decimos, en forma más bien vulgar, que Krushchev fue, a fin de cuentas, una buena cosa, lo que queremos decir es que en conjunto fue bueno que él estuviera a cargo de Rusia. A veces, cuando escuchamos noticias acerca del rescate de algunas personas en algún gran desastre, proferimos una única palabra, ¡Bien! como una exclamación con el mismo sentido. Obviamente los utilitaristas "agatísticos", como Brentano y Moore, hablan de bueno de acuerdo a este uso no cualificado cuando dicen que siempre debemos llevar a cabo aquel acto que en balance produzca mayor bien o menor mal. Hemos regresado así, después de una larga pero necesaria digresión acerca del valor en general, a un punto de central importancia para la filosofía moral, y debemos preguntar de inmediato cómo se aplica aquí nuestra consideración general del significado de "bueno". No sé si fue el uso de "bueno" en "bueno como un X" o el uso no cualificado el que apareció primero, pero parece claro que los dos usos están muy cercanamente relacionados en todos los lenguajes naturales y que debe ser posible encontrar una consideración general del significado de "bueno" que los abarque a ambos. Desde luego una consideración que cubriera sólo el uso cualificado no sería suficiente para la ética. Porque, a pesar de que los utilitaristas puedan estar equivocados al pensar que los actos deben ser valorados sólo con respecto a sus consecuencias, ciertamente debemos encontrar lugar en alguna parte para la consideración de la bondad (o maldad) de estados de cosas, que es bondad (o maldad, según sea el caso) de la clase no cualificada.

Es un hecho importante acerca del uso cualificado de la palabra "bueno" que conlleva un compromiso del que habla en favor de aquello a lo que aplica la palabra en un enunciado de la forma "Esto es un buen X", y que, desde luego, ésta es la razón por la que la palabra puede ser usada para recomendar cosas y aconsejar a la gente en sus decisiones. Pero hemos visto que este compromiso puede surgir de dos maneras distintas. A veces proviene simplemente de la propia identificación del que habla con un grupo en el cual un cierto interés es universal o casi universal. Cuando hablamos del tiempo soleado como de un buen tiempo, usamos la frase "buen tiempo" como si significara "tiempo de la clase que nos gusta", y es claro para cualquiera que reflexione sobre ello que el llamar bueno a tal tiempo depende de que vivimos en un clima de una cierta clase y tenemos unos ciertos intereses particulares. Si viviéramos en el Sahara no le llamaríamos bueno a tal 
tiempo; y aun en Inglaterra no le llamaríamos bueno si fuésemos granjeros o ingenieros hidráulicos, y el día soleado hubiera estado precedido por muchos otros días similares. Mientras permanezcamos en este nivel de discurso, podremos obtener asentimiento de nuestros oyentes sólo porque nosotros mismos somos normales en el sentido de tener los mismos intereses que la mayoría de nuestra sociedad. Pero cuando hacemos nuestras cualificaciones más precisas, en particular cuando incluimos en ellas referencias explícitas a intereses, la situación cambia, por ejemplo, cuando decimos que el tiempo en agosto fue bueno para la maduración de la cosecha, ya no se supondrá que a nosotros mismos nos fue agradable el brillo del sol. Aún más, si fuésemos propietarios de un cine costero pediríamos constantemente lluvia con la esperanza de que esto incrementara nuestras entradas. Lo que queremos decir es más bien que el tiempo en agosto fue de la clase que se necesita para la maduración de la cosecha y, por tanto, de la clase que nos hubiera gustado si hubiéramos querido obtener una cosecha madura. Ahora bien, en tanto que nuestro compromiso es sólo con un favor condicional, nuestras probabilidades de obtener asentimiento para nuestra afirmación no dependen de ninguna comunidad de intereses entre nosotros y nuestros oyentes. Todo lo que se necesita es que tales oyentes sean racionales y reconozcan la importancia de lo que decimos con respecto a ciertos posibles intereses de los seres humanos. Si el interés en cuestión es tal que un oyente no pueda imaginarse fácilmente a sí mismo compartiéndolo, nuestra afirmación puede ser algo cómica, como cuando, por ejemplo, decimos que una noche oscura es buena para robar; pero seguirá siendo inteligible.

En mi intento de clasificar varios usos cualificados de la palabra "bueno", empecé con ejemplos como "un buen cuchillo" y "un buen plomero", pero no creo que haya ninguna evidencia para mostrar si éstos aparecen antes o después de ejemplos tales como "una buena manzana" y "un buen caballo"; lo que parece más factible es que los hombres hayan usado "bueno" en contextos de ambas clases por mucho tiempo y que la diferencia entre los casos sea simplemente aquella que hay entre asunciones restringidas y amplias de uniformidad. La racionalidad es, por así decirlo, la mínima asunción de uniformidad que podemos hacer en una discusión equitativa con otros. Si pensamos que la gente es incapaz o está poco dispuesta a convenir con nosotros en que en general los cuchillos que pueden cortar son los cuchillos que deben comprarse, entonces no le hablamos, excepto quizá como hablamos a los animales o a los niños muy pequeños. Pero por lo común, podemos asumir identidad de interés y concordancia de actitud sobre una gran cantidad de hechos prácticos $\mathrm{y}$, por esa razón, no vacilamos en hablar a nuestros vecinos acerca de buenas manzanas, buenos caballos, buen tiempo, etc. A pesar de que reconocemos que es concebible que ellos tengan intereses en las manzanas, los caballos y el tiempo, diferentes de los nuestros. Si mi acceso gene- 
ral al problema es correcto, podemos esperar encontrar una situación de alguna manera similar cuando consideremos los usos no-cualificados de bueno.

\section{$\S 3$}

Si un niño dice “¡Bueno!” al escuchar que habrá fresas para la siguiente comida, puede contar usualmente con el asentimiento de todos los presentes que se espera compartan la comida. Fue precisamente en tal situación cuando aprendimos por primera vez el uso de la palabra bueno por sí mismo. En forma similar, si digo "¡Bien!” después de escuchar por el radio el pronóstico de que el tiempo será soleado en los próximos días, no espero escuchar ningún desacuerdo, porque de ordinario no hago tal afirmación a menos de que tenga la suficiente confianza en que nadie estará en desacuerdo. Es más, a veces nuestro uso de la palabra bueno en una forma exclamativa parece no ser sino un gesto de amistad y solidaridad. Supóngase, por ejemplo, que $A$ y $B$ son candidatos rivales para un nombramiento atractivo y que $A$ es mi amigo, pcro $B$ no es más que un conocido. Al escuchar que $A$ ha sido elegido puedo exclamar ¡Bien! en presencia de otras personas más estrechamente relacionadas con $A$ que con $B$; pero con seguridad no exclamaré ¡Bien! en presencia de $B$ o de sus amigos íntimos a menos que quiera ofenderlo. Porque puede asumirse que, aunque piense sinceramente que $A$ es el más apto para el trabajo, no tengo ninguna necesidad de decir nada acerca del asunto, y sería innecesariamente provocativo si expresara mi juicio a personas que, de alguna manera, están descontentas por el resultado. Se puede pensar quizá que éstos son usos triviales de la palabra "bueno" y que no son dignos de la atención de un filósofo. Pero creo que son interesantes porque muestran que tanto en nuestros comentarios acerca de los estados de cosas como en nuestros comentarios acerca de las manzanas, los caballos y el tiempo, nuestro uso de la palabra presupone de hecho frecuentemente una cierta comunidad de intereses entre nosotros y aquellos a quienes hablamos.

Admito que los ejemplos que he presentado hasta ahora no son como los de Brentano o Moore o quizá como los que cualquier otro filósofo de la moral tendría en la mente al hablar acerca del deber de producir el máximo bien, sino que son usos bastante comunes y parece claro que no hay una nítida separación entre ellos y los usos que han interesado a los filósofos de la moral. Me parece que la diferencia es simplemente que en los casos que he citado hasta ahora, nuestro uso de "bueno" implica la asunción de una comunidad de intereses semejante a la que puede existir entre los miembros de cualquier grupo. Por ejemplo, familia, colegio, gremio, nación, mientras que en un enunciado de la clase que Moore consideraría pertinente a una decisión acerca de lo que debemos hacer, nuestro uso de la palabra sólo implica la asunción de una comunidad de intereses tal como la que podemos 
esperar encontrar entre todos los sujetos razonables. Esta diferencia está conectada muy naturalmente con el contexto. Si el director de una compañía por acciones dice en su informe a los accionistas: "lo más que podemos esperar para el próximo año es mantener el presente nivel de ventas", todos ellos entienden que les está hablando acerca de la manera en la que sus intereses especiales podrian ser afectados. Por otro lado, cuando un moralista dice que los hombres deben producir el mejor estado de cosas que puedan, es claro que se refiere a ellos más como hombres que como accionistas, y que no asume ningún interés común excepto aquel que se espera compartan todos los hombres. Desde luego no es imposible que el director de una compañía por acciones se refiriese a los accionistas en tanto que hombres y los invitara a aprobar un acto al que no le encontraran ninguna ventaja especial en tanto accionistas; pero si lo hiciesen, algo en la forma en la que presentara el caso mostraría el cambio. La distinción sobre la que he llamado la atención es descrita algunas veces como aquella que media entre lo bueno para ciertas personas y lo bueno en absoluto. Mediante esta terminología puedo aclarar mi punto de vista diciendo que lo que llamamos bueno en absoluto es concebido aún por nosotros como bueno para los hombres qua hombres. Aun aqui hay una asunción de interés común, aunque no de la clase que es concebible que faltara en personas con las que podríamos conversar satisfactoriamente. Pero con el fin de evitar confusiones es necesario decir aquí algo más para la clarificación de la noción del interés común.

A veces, cuando hablamos del interés común de cierto número de personas, sólo queremos decir un interés con respecto al cual son semejantes aunque una pueda conseguir lo que quiere sin que otra lo consiga. En este sentido, el interés por conseguir el sustento puede llamarse común a todos los hombres, pero no hay rảzón para suponer que este interés los lleve siempre a colaborar, puesto que puede ser que, en ciertas circunstancias, uno solo pueda conseguir comida para sí tomando lo que otros desean. En este caso tal vez fuera más sensato hablar de intereses similares. Sin embargo, sea como fuere, este caso debe distinguirse seguramente del caso en el cual cierto número de personas se interesa por algo en tal forma que no es concebible que entren en conflicto una con otra en relación con ese interés. Así los miembros de un equipo puede decirse que tienen un interés común en el éxito del equipo y la palabra "común" parece aquí más apropiada porque se la aplica a un deseo que no puede ser satisfecho con respecto a uno y no al otro. De hecho, realmente tenemos dos usos de la palabra "interés". En el primero pensamos sólo acerca de un interés en el sentido de una condición psíquica como el hambre. En el segundo pensamos también acerca de un interés en el sentido de algo que un hombre desearía haber realizado, el objeto de un deseo. A veces un interés que es común en el primer sentido sólo puede conducir a la gente a comprometerse en empresas con respecto a las cuales 
tendrán un interés común en el segundo sentido de la frase. Si cierto número de personas que buscan todas un provecho semejante, cada una por sí misma, forma una compañia por acciones, tendrán pues un interés común en el éxito de la compañía, y para que tal situación se presente no es ni siquiera necesario que deba haber cooperación. Si cierto número de campesinos cultiva la tierra, cada uno para su propio beneficio, pueden tener un interés común en que llueva en una determinada época del año. Ahora bien, es evidente que todos los hombres tienen muchos intereses que son intereses comunes sólo en el primer sentido, y también algunos que sólo son intereses comunes en el segundo sentido. Cada uno de nosotros quiere comida para si mismo $y$, en consecuencia, tenemos un interés común en conservar la atmósfera libre de sustancias pelígrosas como el estroncio go. Con mucha frecuencia, cuando argumentamos acerca de lo bueno para el hombre en tanto que hombre tratamos de llamar la atención sobre intereses comunes semejantes al de mi ejemplo, y a veces lo logramos; así como los directores de una compañía por acciones pueden conseguir el asentimiento de los accionistas acerca de lo bueno para ellos en tanto que accionistas. De hecho, hay varias empresas internacionales, por ejemplo, la Organización Mundial de la Salud, que los hombres sostienen primariamente por razones de esta clase. Pero en tal caso nos apoyamos aún en lo que he llamado similitudes de facto entre los hombres, esto es, similitudes que podemos confiar en encontrar en casi todos los hombres, pero que sin embargo podemos no encontrar en algunos casos raros; y así no hemoṣ alcanzado aún el límite de abstracción de los intereses especiales. Surpóngase que hay alguien que sabe que morirá con seguridad muy pronto y no puede comer mucho más, pero está todavía en posibilidad de seguir una discusión acerca del desarme nuclear y quizá está aún en posibilidad de ejercer una influencia decisiva en los debates políticos de su propio pais. Es evidente que tal hombre no puede ser conmovido por la consideración de un interés común de la clase que ha hecho notar o, más bien, no por la consideración del lugar que tal interés tiene y tendrá en su propia vida. Esto, sin embargo, no quiere decir que tal persona no pueda ser conmovida por ninguna consideración $y$, en particular, que no tenga ningún interés humano común al que podamos apelar. En última instancia, cuando ya no se puede contar con una comunidad de intereses como aquella que unifica a los hombres en grupos especiales y los lleva a usar la palabra "bueno" dentro de esos. grupos de la manera que he indicado, hay aún un interés que debemos asumir si vamos a tener una discusión moral y es un interés compasivo en la: satisfacción de los deseos de otros seres sensibles.

Si digo que un incremento de estroncio go en la atmósfera es una cosa mala y que sería bueno eliminarlo, la persona a quien hablo puede muy bien preguntar "¿por qué?" Porque el juicio que hago se basa en una información que puede no ser accesible a todos. Supóngase entonces que explico to me- 
jor que puedo los hechos científicos acerca de la absorción de estroncio en las plantas comestibles, los efectos que éste tiene en los cuerpos de la gente que lo ingiere y que la persona a quien hablo dice: "Sí, entiendo todo eso, pero el estroncio go no me puede afectar, ya que voy a morir pronto y los efectos de los que usted habla no se presentarán, en todo caso, hasta dentro de unos años." Es evidente que ha presentado un argumento del tipo que sería conclusivo en contra de muchas clases de propaganda. Si un comerciante de carbón le escribe, durante el verano, a un cliente sugiriéndole que sería bueno comprar cierta cantidad de carbón antes de que los precios alcancen su nivel de invierno, el cliente tiene una réplica absolutamente concluyente si sabe que morirá antes del invierno. Pero en el caso que estamos considerando no debemos aceptar tal réplica. Debemos perseverar y decir "el estroncio go puede no afectar su salud, pero con toda seguridad le interesa lo que va a pasarles a los otros". Si en este punto la persona a quien hablamos dice "No, me da igual, après moi le déluge", no podemos llevar el argumento más adelante, pero no renunciamos pues pensamos que el hombre que dice esto ha hecho una aceptable renuncia de membrecía al grupo al que otros han asumido, erróneamente, que pertenece. Hay muchas ocasiones en las que es permisible usar la famosa observación de Samuel Goldwyn: "inclúyanme fuera", pero ésta no es una de ellas. Si un hombre dice con aparente sinceridad que no le interesa la satisfacción de ningún deseo excepto el suyo propio, lo consideramos loco en un sentido especial. Ya que, en efecto, muestra que no es capaz de entender el uso absoluto de "bueno" y "malo" en discusiones morales.

Aquí tenemos un paralelo con nuestro uso cualificado de la palabra "bueno" en "un buen cuchillo". Justamente como allí aplicamos a veces la palabra a alguna cosa sin comprometernos a ser miembros de ningún grupo -a no ser el de los hombres racionales-, así aquí. Sin embargo, hay aquí una diferencia interesante. Cuando un hombre dice que un cuchillo no puede ser bueno a menos que corte, sostiene que cualquiera que esté en desacuerdo es irracional en el sentido de ser incapaz de apreciar razones teóricas de una clase obvia. Cuando, sin embargo, un hombre dice que un hecho es malo en tanto causa gran sufrimiento, sostiene que cualquiera que no esté de acuerdo es irracional en el sentido de ser incapaz de apreciar las muy obvias razones para adoptar una actitud. No es un accidente, claro está, que hablemos de razones en ambos contextos; porque en cada uno de ellos, una razón es algo que un hombre presenta a otro en un argumento con el fin de formar o cambiar el punto de vista del otro. Pero hay una diferencia reconocible entre el sentido en el que la verdad de las premisas de un silogismo es una razón para aceptar la conclusión, y el sentido en que el hecho de que un temblor haya causado sufrimiento es una razón para lamentar que haya sucedido. Cuando hablamos de racionalidad, tenemos con frecuencia en men- 
te la capacidad de reconocer razones de ambas clases, pero hay una tendencia a conservar "racionalidad" con el significado de capacidad para reconocer razones de la primera clase, mientras que usamos "razonabilidad" para mentar asimismo una cualidad moral. Quizá la explicación es que la forma de la palabra "razonable" sugiere apertura al razonamiento, es decir, disposición para considerar las razones propuestas por otra gente, mientras que "racional" no está tan obviamente conectada con la vida social.

El mismo punto puede ser formulado de otra manera. Si digo "un cuchillo que no corta es un mal cuchillo", mi enunciado es analítico, no porque la frase "mal cuchillo" haya adquirido el llamado significado descriptivo de "cuchillo que no corta", sino, más bien, porque el enunciado total puede ser parafraseado en la forma "un instrumento para cortar que no corta es tal que una persona razonable no desearía tenerlo si necesitara un instrumento para cortar". De manera semejante digo "un estado de cosas que implica gran sufrimiento es, como tal, un mal estado de cosas", mi afirmación es analítica, no porque "malo" signifique "doloroso", como podría haber dicho Bentham, sino porque el todo es equivalente a "un estado de cosas que implica un gran sufrimiento es tal que una persona razonable no desearía verse envuelto en él, siéndole indiferente en igualdad de circunstancias". Pero en el segundo caso, el sentido de "razonable" requerido para la paráfrasis es el sentido fuerte señalado en el último párrafo. Porque lo que aqui importa no es meramente la capacidad para evitar la inconsistencia sino la apertura a ser alterado justo por consideraciones como la presencia de sufrimiento.

En este punto un prescriptivista podría objetar de la siguiente manera: "usted empezó diciendo que la bondad y la maldad iban a ser definidas con referencia a lo que agrada y desagrada a los hombres normales. Ahora, admite que en los casos realmente importantes de juicio moral, distintos de la cháchara social, le da a la palabra 'normal' un sentido normativo que la hace equivalente a 'razonable', y ha llegado al extremo de admitir que en vez de decidir si algo es bueno recolectando los votos de la gente que parece ser razonable, usted decide si un hombre es o no razonable tomando en cuenta si está de acuerdo o no con usted en tener ciertas preferencias y desavenencias a las que usted otorga gran importancia. La conclusión de su larga jerigonza es por tanto que la palabra 'bueno' se usa en contextos morales en vez de recomendación o prescripción. ¿Por qué no decirlo abiertamente?" Frente a esto debo repetir primero una objeción contra el prescriptivismo hecha por varios otros críticos, esto es, que la palabra "bueno" aparece frecuentemente, aun en el discurso moral, donde no puede decirse con propiedad que se use en vez de recomendación y ni siquiera que tenga un significado puramente descriptivo, si suponemos que un significado descriptivo es algo diferente al que tiene cuando se usa en juicios de valor. Cuando un hombre se pregunta seriamente a sí mismo si un cierto estado de cosas sería bueno, no está reco- 
mendando el estado de cosas, ni se pregunta si lo recomienda, porque ex hipothesi no lo ha decidido aún. En segundo lugar, debo tratar de explicar que el sentido normativo de "normal" tiene una conexión cercana al sentido estadístico y nunca puede ser del todo independiente. He tratado de ilustrar antes esta tesis al discutir la visión de color y la ceguera de color y trataré de aclararla ahora por medio de la referencia a las nociones de salud mental y locura, que están conectadas mucho más de cerca con la clase de normalidad que me interesa.

Es perfectamente posible que un hombre piense que todo el mundo se ha vuelto loco y esté en lo cierto al pensarlo así. Es decir, no hay un absurdo en la suposición de que todos los demás se hayan vuelto locos. Pero es imposible que un hombre piense correctamente que él es el único ser cuerdo que ha existido, pues esta suposición anula la posibilidad de que haya aprendido a distinguir entre salud mental y locura. A pesar de que nuestra noción de salud mental es normativa, en un cierto sentido, porque implica un estándar que la gente puede no alcanzar, está muy firmemente relacionada con nuestra noción de lo que ha sido la costumbre entre la gran mayoría de las personas que ha tenido una parte activa en la sociedad y la mantiene. Los hombres son animales sociales, no sólo en el sentido de ser, como las hormigas, criaturas que de hecho forman sociedades cuando tienen la oportunidad, sino también en el sentido más fundamental de ser animales que no pueden procurarse por si mismos la clase de vida distintiva de sus especies; y la locura es básicamente la incapacidad para tomar parte en la sociedad. No quiero decir con esto que los locos sean insociables, esto es, estén poco dispuestos a asociarse con otros, aunque éste sea con mucha frecuencia el caso, sino más bien que no están abiertos a la influencia del mundo y en particular de los otros hombres en la forma necesaria para la conservación de la sociedad. Cualquiera puede tener una extraordinaria experiencia sensorial cuando está extremadamente cansado o bajo la influencia de una droga, pero la mayoría de nosotros somos capaces de reconocer tales experiencias como lo que son, porque tenemos un deseo de adaptarnos a la realidad por el experimento y conservándonos en relación con otra gente; en resumen, nosotros tenemos una mente abierta y crítica. Por otro lado, los locos no pueden distinguir entre lo ordinario y lo extraordinario porque están separados de la clase de vida que nos permite, al resto de nosotros, descubrir la diferencia. Lo mismo vale aún más obviamente con respecto a las actitudes emocionales.

Si estoy en lo correcto, esta razonabilidad por referencia a la cual debemos definir la bondad o la maldad de un estado de cosas es algo sin lo cual no podría haber una sociedad humana, y por esta razón no puede ser un molde mental que uno de nosotros adopte por primera vez al hacer un juicio de valor. Aquellos que sostienen la teoría prescriptivista de los valores y proclaman lo que llaman la autonomía de la ética, hablan a veces como si 
pensaran que cualquier hombre puede en cualquier momento prescribir o recomendar una acción bastante diferente de cualquier cosa que él o cualquier otra gente haya prescrito antes, y que su juicio será moral siempre y cuando esté comprometido a favor de todas las acciones semejantes a ella en algún aspecto específico. Algunos existencialistas parecen ir aún más allá y omiten la estipulación acerca de recomendaciones generales. Aun la menos extrema de estas dos posiciones me parece ser una doctrina del caos, más que de la autonomía. Naturalmente no deseo negar que pueda haber alli innovaciones morales sorprendentes, pero sostengo que los hombres no podrían ni siquiera entender lo que quiere decir un innovador moral con sus palabras a menos que puedan pensar que su enseñanza es un desarrollo o refinamiento de lo que ya han aceptado. La sociedad no es sólo una batalla de personalidades en la que los hombres se dictan prescripciones unos a otros y la victoria toca a la personalidad más fuerte. Sin duda, a veces personas como Nietzsche y Hitler tienen éxito de hecho e imponen códigos morales revolucionarios a personas que son susceptibles de ser dominadas por megalomaníacos, pero éste no es el modelo ordinario de desarrollo moral, y aun en estos casos hay justo el contacto suficiente con la tradición para que sea posible que los conversos piensen que la novedad es algo que pueden presentar de modo adecuado con los antigucs términos de valor. Nietzsche y Hitler, por ejemplo, jugaron mucho con palabras como "heroico", que tiene asociaciones morales, pero que también se puede usar para sugerir habilidad marcial, como la de los guerreros homéricos o la de los vikingos. Cuando un reformador moral recomienda algo a sus oyentes mediante el uso de palabras como "bueno", lo que hace en efecto es decir que la cosa que recomienda es algo a cuyo favor estarían todos ellos si fueran lo bastante razonables, esto es, lo suficientemente cuidadosos y cabales en esa clase de reflexión imparcial acerca de los deseos a las que están ya obligados por ser miembros de una sociedad humana y, en particular, por su uso de la palabra "bueno" en forma absoluta. Puesto que ex hipothesi no están aún a favor de lo que él les recomienda, su afirmación puede ser tomada como una pretensión de haberlos aventajado en razonabilidad, y corre por lo tanto el riesgo de hacer caer sobre sí mismo una acusación de arrogancia. En su propia defensa contra esta acusación, el reformador moral sólo puede argüir que lo que ha dicho está abierto a la verificación de otros, si éstos adoptan el entrenamiento apropiado para hacer de sí mismos jueces confiables de lo bueno en sentido absoluto. En otras palabras, pretende que su novedad es un descubrimiento, no una creación. Aun Nietzsche y Hitler optaron por este camino en su prédica (qué otra cosa podían hacer), a pesar de que en el cuerpo de su enseñanza no es consistente con las implicaciones de su pretensión. En el pasado, los reformadores politicos ingleses trataron con frecuencia de presentar sus reformas como meras aclaraciones de lo contenido en la ley común y en la antigua constitución 
del país. Esta manera de pensar está relacionada, sin duda, con un grado de autosatisfacción nacional sólo igualado por el de los chinos, y me atrevería a decir que nos ha llevado a falsificaciones de la historia, así como a algunas ventajas prácticas. Pero la humanidad como un todo, está autorizada a sostener esta teoría conservadora del progreso moral, porque ninguna otra puede dar una respuesta satisfactoria a las transacciones lingüisticas en las que se presentan las reformas morales. Aquellos que piensan que esto excluye el progreso ignoran la evidencia de la historia, y lo que ofrecen a cambio es una teoría que no deja lugar a ningún otro argumento acerca de los valores que no sea el llamar la atención sobre las inconsistencias de los oponentes, si éstos han caído en alguna.

Lo que he dicho en esta sección de mi artículo acerca de la razonabilidad y sus relaciones con nuestras nociones de valor es algo semejante a lo que dice Aristóteles en su Ética Nicomaquea acerca de la phrónesis. Pues insiste en que ningún hombre puede adquirir esta excelencia intelectual (como él la describe), excepto viviendo en una sociedad, y también que tal excelencia es necesaria aun para la organización de la sociedad más simple, como la familia. Algunas veces los críticos han objetado que hay un círculo en $s u$ argumento, y que él mismo parece reconocer una dificultad cuando admite que no puede haber un principio absoluto de vida social, que considera esencial al hombre, a no ser a través de la acción de un legislador divino que posea la phrónesis sin haberla adquirido en la vida social. Pero el problema desaparece si damos un paso más allá del que Aristóteles mismo dio al pensar al hombre como producto de la evolución. Pues es fácil ver que en la especie humana, que no es físicamente muy fuerte en comparación con los leones y los tigres, cualquier pequeño principio de capacidad para la organización social sería altamente favorecido por la selección natural. Hay sin embargo dos diferencias entre lo que he dicho acerca de la razonabilidad y la doctrina aristotélica de la phrónesis. Primero he usado la noción de elección de un hombre razonable para una definición de la bondad, más que para una definición de la acción correcta como lo hace Aristóteles. Lo hice así, porque estoy de acuerdo con Brentano y Moore en tomar la noción de bondad como la más fundamental en la moralidad racional. En segundo lugar, he destacado más que Aristóteles la importancia de la consideración armónica hacia los deseos de otros. A pesar de que relaciona en forma muy cercana la phrónesis con una capacidad para la vida social y la amistad, parece pensar primariamente en ella como una racionalidad en el sentido de una capacidad para hacer una elección sabia de medios a fines. Literalmente el adjetivo phrónimos quiere decir "atento", pero en el griego común y corriente no posee la sugestión de preocupación por el bienestar de los otros que se aplica a nuestra palabra. La diferencia es importante y digna de subrayarse, porque la razonabilidad en el sentido de disposición para ser movido por la 
consideración de los deseos de otros, nos entrega el único estándar posible para el uso de "bueno" en una forma absoluta. En algunas circunstancias, cualesquiera otros intereses de los hombres pueden producir un choque. Quizá la forma más facil para aclarar este punto sea preguntar qué necesitaríamos encontrar en seres de otro planeta para poder llegar alguna vez a un entendimiento con ellos acerca de cuestiones morales. Es evidente que ellos pueden tener apetitos e intereses muy diferentes a cualquiera de los nuestros, pero esto no importaría si fueran a) racionales en el sentido de ser capaces de seguir argumentos teóricos y $b$ ) estar dispuestos a ser movidos por la consideración de los deseos de otros. Pero éstas son precisamente las capacidades cubiertas por la palabra "razonable".

WiLliam Kneale

[Trad. de Salma Saab y Raúl Quesada]

UNIVERSIDAD DE OXFORD 